\title{
ISO far-infrared observations of the high-latitude cloud L1642 ${ }^{\star, \star \star}$
}

\section{Correlated variations of far-infrared emissivity and temperature of "classical large" dust particles}

\author{
K. Lehtinen ${ }^{1}$, M. Juvela ${ }^{1}$, K. Mattila ${ }^{1}$, D. Lemke ${ }^{3}$, and D. Russeil ${ }^{1,2}$ \\ ${ }^{1}$ Observatory, Tähtitorninmäki, PO Box 14, 00014, University of Helsinki, Finland \\ e-mail: kimmo.lehtinen@helsinki.fi \\ 2 Laboratoire d'Astrophysique de Marseille, 2 place Le Verrier, 13004 Marseille, France \\ 3 Max-Planck-Institut für Astronomie, Königstuhl 17, 69117 Heidelberg, Germany
}

Received 28 November 2006 / Accepted 30 December 2006

\section{ABSTRACT}

\begin{abstract}
Aims. Our aim is to compare the infrared properties of big, "classical" dust grains with visual extinction in the cloud L1642. In particular, we study the differences in grain emissivity between diffuse and dense regions in the cloud.

Methods. The far-infrared properties of dust are based on large-scale $100 \mu \mathrm{m}$ and $200 \mu \mathrm{m}$ maps. Extinction through the cloud was derived by using the star count method in the $B$ - and $I$-bands, and colour excess method in the $J, H$, and $K_{\mathrm{s}}$ bands. Radiative transfer calculations were used to study the effects of increasing absorption cross-section on the far-infrared emission and dust temperature. Results. Dust emissivity, measured by the ratio of far-infrared optical depth to visual extinction, $\tau($ far-IR $) / A_{\mathrm{V}}$, increases with decreasing dust temperature in L1642. There is about a two-fold increase in emissivity over the dust temperature range of $19 \mathrm{~K}-14 \mathrm{~K}$. Radiative transfer calculations show that, in order to explain the observed decrease of dust temperature towards the centre of L1642, an increase of absorption cross-section of dust at far-IR is necessary. This temperature decrease cannot be explained solely by the attenuation of interstellar radiation field. Increased absorption cross-section also manifests itself as an increased emissivity. We find that, due to temperature effects, the apparent value of optical depth $\tau_{\text {app }}$ (far-IR), derived from $100 \mu \mathrm{m}$ and $200 \mu \mathrm{m}$ intensities, is always lower than the true optical depth.
\end{abstract}

Key words. ISM: individual objects: Lynds 1642 - ISM: clouds - ISM: dust, extinction - infrared: ISM

\section{Introduction}

Recent observations have indicated that the far-IR emissivity (measured by the ratio $\tau\left(\right.$ far-IR) $\left./ A_{\mathrm{V}}\right)$ increases with decreasing temperature (Cambrésy et al. 2001; del Burgo et al. 2003; Stepnik et al. 2003; Kramer et al. 2003). Probable reasons for increased emissivity are coagulation of dust particles into large, fluffy aggregates and formation of ices on grain surfaces in dense regions (Bazell \& Dwek 1990; Preibisch et al. 1993; Ossenkopf \& Henning 1994; Dwek 1997; Cambrésy et al. 2001 and references therein). Variations in dust's optical properties are visible even on a scale of the galactic anticentre hemisphere, anywhere where $A_{\mathrm{V}} \gtrsim 1 \mathrm{mag}$ (Cambresy et al. 2005). Del Burgo \& Laureijs (2005) used observations of the Taurus molecular cloud TMC- 2 between $60 \mu \mathrm{m}$ and $200 \mu \mathrm{m}$ to separate the emission into cold and warm components. They found that far-IR emissivity of the cold component is a few times larger than that of the diffuse interstellar medium, and that the change in the properties of the big dust particles takes place at intermediate densities of $n\left(\mathrm{H}_{2}\right) \approx 10^{3} \mathrm{~cm}^{-3}$. They favoured grain-grain coagulation over gas accretion as the reason for enhanced emissivity because the latter process produces an increase in emissivity by a factor of

* Based on observations with ISO, an ESA project with instruments funded by ESA Member States (especially the PI countries: France, Germany, The Netherlands, and the UK) and with the participation of ISAS and NASA.

$\star \star$ Appendices A-D are only available in electronic form at http://www . aanda. org about two at most, less than observed by del Burgo \& Laureijs. Also, Stepnik et al. (2003) suggest that grain-grain coagulation is the reason for changes in dust properties in dense regions.

For the introduction of the high galactic latitude dark cloud L1642, we refer to Lehtinen et al. (2004, Paper I) and references therein. Paper I was based on ISOPHOT observations in the farinfrared, and we made the following conclusions:

- Based on the $200 \mu$ m optical depth map, the cloud was divided into three regions, called A (consists of two cloudlets, A1 and A2), B, and C (see Fig. 1).

- Region $\mathrm{B}$, the one with the highest dust column density, $A_{\mathrm{V}} \approx 8 \mathrm{mag}$, is related to a temperature minimum, $T_{\mathrm{d}} \approx$ $13.8 \mathrm{~K}$ for a $v^{2}$ emissivity.

- This region is close to being gravitationally bound, while the others are not.

- The lower dust temperature in region B cannot be explained solely by the dust attenuation of the general interstellar radiation field, but changes in the properties of dust particles are required.

In this paper we will compare the infrared properties of big grains with visual extinction. By comparing far-IR emission data with optical extinction data, we can study the infrared and optical properties of dust grains. In particular, combination of reliable dust temperature, far-IR optical depth, and visual extinction data enables us to study the differences in grain emissivity between diffuse and dense regions in the cloud. 


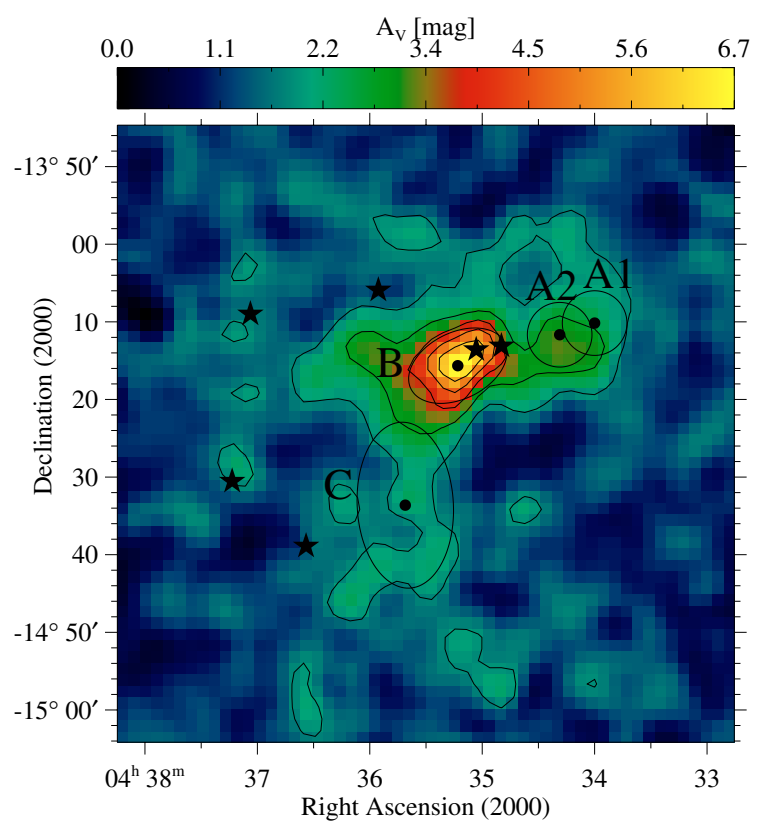

Fig. 1. Visual extinction through L1642, derived using $J, H$, and $K_{\mathrm{s}}$ data from the 2MASS archive, smoothing the individual extinctions of stars with a Gaussian with $F W M H=4.5^{\prime}$. The IRAS sources, marked with asterisks, are from right to left: IRAS 04325-1419, 04327-1419, 04336-1412, 04342-1444, 04347-1415, and 04349-1436. The FWHM sizes of the regions A1, A2, B, and C as derived by Lehtinen et al. (Paper I) from $200 \mu \mathrm{m}$ optical depth data are shown as ellipses and circles. The contours are at 1.5, 2.5, 3.5, 4.5, and $5.5 \mathrm{mag}$.

\section{Data analysis}

For the data reduction of the ISO data we refer to Paper I. Extinction through L1642 has been determined via star count method at optical, and colour excess method at near-IR wavelengths. Near-IR $J, H$, and $K_{\mathrm{s}}$ band data were extracted from the 2MASS ( 2 Micron All Sky Survey) archive. The two binary $T$ Tauri stars near the centre of the cloud were excluded from the 2MASS data.

\subsection{B- and I-band extinction from starcounts}

Details of the starcounts are given in Appendix A. We state here the conclusion that the extinction law in L1642 is similar to that of the diffuse interstellar matter, corresponding to $R_{\mathrm{V}}=A_{\mathrm{V}} / E_{B-V}=3.1$ (see Fig. A.2).

\subsection{Extinction from near-IR colour excess}

In order to derive extinctions from near-IR colour excesses of stars visible through L1642, we applied the optimized multiband technique of Lombardi \& Alves (2001) called NICER, which is a generalisation of the traditional colour excess method using only data of two bands. Our data for L1642 comprise $J, H$, and $K_{\mathrm{s}}$ band magnitudes from the 2MASS archive. The surface density of stars detected in all three bands is constant throughout the map, with an average of about 0.9 stars $/ \square^{\prime}$. The details of our extinction determination are given in Appendix B. The resulting visual extinction map, weighted with a $F W H M=4.5^{\prime}$ Gaussian, is shown in Fig. 1. Region B has a maximum visual extinction of $\sim 6.7 \mathrm{mag}$. At $F W H M=3^{\prime}$ resolution the maximum extinction rises to $\sim 8.2 \mathrm{mag}$. Region $\mathrm{A}$ is seen as a local maximum, but the angular resolution is not high enough to resolve the clumps A1 and A2. In general, the correspondence between visual extinction on the one side and the $200 \mu \mathrm{m}$ surface brightness and optical depth maps (see Paper I) on the other is very good. There are, however, some differences. The $200 \mu \mathrm{m}$ surface brightness and optical depth of regions $\mathrm{A}$ and $\mathrm{C}$ are about equal. However, the maximum extinction of region $\mathrm{C}(\sim 2.0 \mathrm{mag})$ is clearly lower in region $\mathrm{A}(\sim 3.0 \mathrm{mag})$. Furthermore, the extinction over region $\mathrm{C}$ does not have a similar regular distribution with a single maximum as the far-IR surface brightness and optical depth maps do.

There are differences between the USNO-based and 2MASS-based extinctions. First, the maximum visual extinction derived from star counts is about half of the maximum derived from near-IR colour excesses. This can at least partly be explained by the lower resolution and lower penetration power of the star count map. Second, the relative values of extinction in regions $\mathrm{A}$ and $\mathrm{C}$ are different; in the USNO-based extinctionmap, region $\mathrm{C}$ has a larger extinction than region $\mathrm{A}$, but in the 2MASS-based map the situation is reversed. The reason for this is unknown. For all subsequent analyses we will use the 2MASSbased extinction map.

In the following data analysis of this paper, we used only those datapoints for which the signal-to-noise ratio of $A_{\mathrm{V}}$ is greater than two. In practice, this limit sets the lowest $A_{\mathrm{V}}$ to about $0.5 \mathrm{mag}$.

\subsection{Zero-level of extinction and far-IR intensity}

When deriving the emissivity of dust, it is important to avoid the biases caused by improper determination of zero level of extinction and far-IR emission. We have determined the zero levels differently for the interclump dust and the dense regions.

\subsubsection{Interclump dust}

We define the "interclump dust" as the areas in the $200 \mu \mathrm{m}$ map that are outside the regions $\mathrm{A} / \mathrm{B} / \mathrm{C}$ and that have $A_{\mathrm{V}}<2.0$ mag. We expect that interclump dust is low-density material located around the denser regions of L1642 and extending beyond our $200 \mu \mathrm{m}$ map. For interclump dust the zero-level has to be determined at locations sufficiently far away from L1642, outside our $200 \mu \mathrm{m}$ map. Fortunately, in the context of another project, we observed several positions in the vicinity of L1642 with ISO at $200 \mu \mathrm{m}$. Here we utilize the six positions with the lowest $200 \mu \mathrm{m}$ intensity values, located about $2.5^{\circ}$ southwest of the centre of L1642 (TDT, target dedicated time numbers 85500550-55).

The $100 \mu \mathrm{m}$ intensities at the same positions were derived from IRAS/ISSA data. To obtain sufficiently low noise for these zero-level positions in the $A_{\mathrm{V}}$ maps, we used a Gaussian with $F W H M=12^{\prime}$ as a smoothing function, giving about $0.12 \mathrm{mag}$ standard deviation per map pixel. In these six zero-level positions, the value of $I(100 \mu \mathrm{m})=3.4 \pm 0.6 \mathrm{MJy} \mathrm{sr}^{-1}, I(200 \mu \mathrm{m})=$ $4.8 \pm 0.6 \mathrm{MJy} \mathrm{sr}^{-1}$, and $A_{\mathrm{V}}=0.14 \pm 0.11 \mathrm{mag}$ (the given uncertainty is the standard deviation of the parameter). The mean values and $1-\sigma$ deviations of $A_{\mathrm{V}}, I(100 \mu \mathrm{m})$, and $I(200 \mu \mathrm{m})$ within each region are given in Cols. 2-4 of Table 1, after the zerolevels of the interclump dust have been subtracted.

\subsubsection{Regions $A, B$, and $C$}

We consider the regions $\mathrm{A} / \mathrm{B} / \mathrm{C}$ as separate clumps that are seen in excess over the general background of L1642. The zero-levels of regions $\mathrm{A} / \mathrm{B} / \mathrm{C}$ were determined in the immediate surroundings of each of the regions. In this way, we measured emission 
Table 1. Values of parameters in L1642, in other clouds and in models. For a description of the different regions of L1642 see Sect. 2.3. All the data of the cloud L1642 apply to $4.5^{\prime}$ resolution, except columns 4 and 6 which are for $3.5^{\prime}$ resolution. For a description of methods A and B see Sect. 3.2.

\begin{tabular}{|c|c|c|c|c|c|}
\hline Region & $\begin{array}{c}\left\langle A_{\mathrm{V}}\right\rangle \\
{[\mathrm{mag}]}\end{array}$ & $\begin{array}{c}\langle I(100 \mu \mathrm{m})\rangle \\
{\left[\mathrm{MJy} \mathrm{sr}^{-1}\right]}\end{array}$ & $\begin{array}{c}\langle I(200 \mu \mathrm{m})\rangle \\
{\left[\mathrm{MJy} \mathrm{sr}^{-1}\right]}\end{array}$ & $\begin{array}{c}\text { Slope of } \\
I(100 \mu \mathrm{m}) \mathrm{vs} . A_{\mathrm{V}} \\
{\left[\mathrm{MJy} \mathrm{sr}^{-1} \mathrm{mag}^{-1}\right]}\end{array}$ & $\begin{array}{c}\text { Slope of } \\
I(200 \mu \mathrm{m}) \mathrm{vs} . A_{\mathrm{V}} \\
{\left[\mathrm{MJy} \mathrm{sr}^{-1} \mathrm{mag}^{-1}\right]}\end{array}$ \\
\hline 1 & 2 & 3 & 4 & 5 & 6 \\
\hline L1642 A & $2.1 \pm 0.6$ & $14 \pm 1$ & $34 \pm 3$ & $1.6 \pm 0.2$ & $4.2 \pm 0.4$ \\
\hline L1642 B & $4.3 \pm 1.1$ & $16 \pm 1$ & $52 \pm 6$ & $0.8 \pm 0.1$ & $4.5 \pm 0.3$ \\
\hline L1642 C & $1.5 \pm 0.2$ & $16 \pm 2$ & $35 \pm 3$ & - & - \\
\hline L1642 interclump dust & $1.7 \pm 0.1$ & $12 \pm 2$ & $28 \pm 3$ & $6.7 \pm 1.6^{\dagger}$ & $17.7 \pm 1.2$ \\
\hline G300.2-16.8 ON1 [1] & $1.8 \pm 0.2$ & & 17 & & $9.2 \pm 2.1$ \\
\hline G300.2-16.8 ON2 [1] & $1.9 \pm 0.2$ & & 21 & & $11.0 \pm 2.5$ \\
\hline G300.2-16.8 ON3 [1] & $2.9 \pm 0.2$ & & 35 & & $12.1 \pm 2.6$ \\
\hline \multirow[t]{2}{*}{ Region } & $\begin{array}{l}T_{\text {dust }} \\
{[\mathrm{K}]}\end{array}$ & $\begin{array}{c}\langle\tau(200 \mu \mathrm{m})\rangle \\
{\left[10^{-4}\right]}\end{array}$ & $\begin{array}{c}\tau(200 \mu \mathrm{m}) / A_{\mathrm{V}} \\
{\left[10^{-4} \mathrm{mag}^{-1}\right]}\end{array}$ & $\begin{array}{c}\sigma^{\mathrm{H}}(200 \mu \mathrm{m}) \\
{\left[\mathrm{cm}^{2} \mathrm{H}-\mathrm{atom}^{-1}\right]} \\
{\left[10^{-25}\right]}\end{array}$ & \\
\hline & 7 & 8 & 9 & 10 & \\
\hline L1642 A (method A) & $16.3 \pm 0.6$ & $1.3 \pm 0.6$ & $1.3 \pm 0.5$ & $0.7 \pm 0.3$ & \\
\hline L1642 A (method B) & $15.9 \pm 0.2$ & - & $0.8 \pm 0.1$ & $0.4 \pm 0.1$ & \\
\hline L1642 B (method A) & $14.2 \pm 0.6$ & $4.9 \pm 2.5$ & $2.2 \pm 0.6$ & $1.2 \pm 0.3$ & \\
\hline L1642 B (method B) & $13.4 \pm 0.3$ & - & $1.9 \pm 0.3$ & $1.0 \pm 0.2$ & \\
\hline L1642 C (method A) & $18.9 \pm 2.6$ & $0.7 \pm 0.5$ & $1.1 \pm 0.8$ & $0.6 \pm 0.4$ & \\
\hline L1642 interclump dust (method A) & $16.3 \pm 0.5$ & $4.0 \pm 1.0$ & $2.4 \pm 0.5^{\ddagger}$ & $1.3 \pm 0.3^{\ddagger}$ & \\
\hline L1642 interclump dust (method B) & $17.1 \pm 1.0$ & - & $2.2 \pm 0.2^{\ddagger}$ & $1.2 \pm 0.1^{\ddagger}$ & \\
\hline G300.2-16.8 ON1 [1] & 17.4 & 1.9 & $1.1 \pm 0.2$ & $0.6 \pm 0.2$ & \\
\hline G300.2-16.8 ON2 [1] & 17.4 & 2.4 & $1.3 \pm 0.2$ & $0.7 \pm 0.1$ & \\
\hline G300.2-16.8 ON3 [1] & 17.5 & 4.0 & $1.4 \pm 0.2$ & $0.7 \pm 0.1$ & \\
\hline TPN [2] & & & 5.3 & 2.5 & \\
\hline G301.2-16.5 [3] & & & & $0.42-0.95$ & \\
\hline L183 [4] & & & $3.4-3.8$ & & \\
\hline L1780 [5] & 15.2 & & 2.6 & 1.4 & \\
\hline Diffuse ISM [6] & & & 3.2 & 1.7 & \\
\hline Diffuse ISM [7] & & & 2.5 & 1.4 & \\
\hline Model [8] & & & & 2.7 & \\
\hline Model [9] & & & 2.9 & 1.5 & \\
\hline Model [10] & & & 4.7 & 2.5 & \\
\hline
\end{tabular}

$\dagger$ The fit has been forced to go through origin

Limited to visual extinction $A_{\mathrm{V}}>1.2 \mathrm{mag}$

[1] Rawlings et al. (2004); [2] Lehtinen et al. (1998); [3] Laureijs et al. (1996); [4] Juvela et al. (2002); [5] Ridderstad et al. (2006); [6] Cambrésy et al. (2001); [7] Dwek et al. (1997); [8] Hildebrand (1983); [9] Li \& Draine (2001b) for diffuse ISM for $R_{\mathrm{V}}=3.1$, interpolated from their Table 6. Value of emissivity $\tau($ far-IR $) / A_{\mathrm{V}}$ has been derived from their value of $\sigma^{\mathrm{H}}\left(\right.$ far-IR) by multiplying it with the ratio $N\left(\mathrm{H}+\mathrm{H}_{2}\right) / A_{\mathrm{V}}=$ $1.87 \times 10^{21} \mathrm{~cm}^{-2} \mathrm{mag}^{-1}$ (see Sect. 3.3); [10] Désert et al. (1990) for diffuse medium. Value of $\sigma^{\mathrm{H}}(\mathrm{far}-\mathrm{IR})$ has been read from their Fig. 3 with about $20 \%$ accuracy. Value of emissivity $\tau($ far-IR $) / A_{\mathrm{V}}$ has been derived from value of $\sigma^{\mathrm{H}}\left(\right.$ far-IR) by multiplying it with the ratio $N\left(\mathrm{H}+\mathrm{H}_{2}\right) / A_{\mathrm{V}}=$ $1.87 \times 10^{21} \mathrm{~cm}^{-2} \mathrm{mag}^{-1}$ (see Sect. 3.3).

and extinction that originates solely in the region in question. Any forground/background emission by interclump dust is in this way eliminated, at least approximately.

For regions $\mathrm{A}$ and $\mathrm{B}$, the value of emissivity can also be derived using the slopes of $I(100 \mu \mathrm{m})$ vs. $I(200 \mu \mathrm{m})$ and $I(200 \mu \mathrm{m})$ vs. $A_{\mathrm{V}}$, which are independent of any background and/or foreground zero-level subtraction. This method is described in Sect. 3.2.

\subsection{Error estimates}

In this paper our main objective is to study the variations in the dust properties over the uniformly mapped L1642 cloud area. The different error sources contributing to the pixel-topixel variations in temperature, far-IR optical depth, and emissivity are listed in Table 2 . They are i) the intrinsic error of each pixel of the $200 \mu \mathrm{m}$ intensity map, as given by the data analysis program PIA (ISOPHOT Interactive Analysis); ii) the pixel-topixel variation in the $100 \mu \mathrm{m}$ IRAS map, estimated to be $5 \%$; iii) the error due to the incomplete flatfield correction of the ISO map, as estimated from the deviations in the intensities of adjacent pixels, in a similar way to the actual flatfield correction estimation (see Paper I); iv) the error due to the non-matching point spread functions of the $100 \mu \mathrm{m}$ IRAS map and the $200 \mu \mathrm{m}$ intensity map that was convolved to the IRAS resolution. (This error was estimated by convolving the ISO map to 5.0' resolution instead of the $4.5^{\prime}$ resolution. As expected, the largest differences, about $1.6 \mathrm{MJy} \mathrm{sr}^{-1}$, are at the positions of the steepest gradients in region $\mathrm{B}$. This error source was applied only to the $200 \mu \mathrm{m}$ map); v) the error of the $A_{\mathrm{V}}$ map as given by the NICER program; vi) the error related to uncertainty of zero-level subtraction of $100 \mu \mathrm{m}$ intensity (see Sect. 2.3); vii) the error related to uncertainty of zero-level subtraction of $200 \mu \mathrm{m}$ intensity; viii) the error related to uncertainty of zero-level subtraction of 
Table 2. Error sources contributing to the pixel-to-pixel variations of temperature, far-IR optical depth, and emissivity. See Sect. 2.4 for explanations.

\begin{tabular}{ccc}
\hline \hline & Error source & Value \\
\hline i) & $\langle\sigma(I(200 \mu \mathrm{m}))\rangle$ & $0.19 \mathrm{MJy} \mathrm{sr}^{-1}$ \\
ii) & $\sigma(I(100 \mu \mathrm{m}))$ & $5 \%$ \\
iii) & $\sigma($ flatfield $)$ & $0.43 \mathrm{MJy} \mathrm{s}^{-1}$ \\
iv) & $\langle\sigma(\mathrm{PSF})\rangle$ & $0.14 \mathrm{MJy} \mathrm{sr}^{-1}$ \\
v) & $\left\langle\sigma\left(A_{\mathrm{V}}\right)\right\rangle$ & $0.32 \mathrm{mag}^{-1}$ \\
vi) & $\langle\sigma($ zero-level $(100 \mu \mathrm{m}))\rangle$ & $0.9 \mathrm{MJy} \mathrm{sr}^{-1}$ \\
vii) & $\langle\sigma($ zero-level $(200 \mu \mathrm{m}))\rangle$ & $2.6 \mathrm{MJy} \mathrm{sr}^{-1}$ \\
viii $)$ & $\left\langle\sigma\left(\right.\right.$ zero-level $\left.\left.\left(A_{\mathrm{V}}\right)\right)\right\rangle$ & $0.43 \mathrm{mag}^{-}$ \\
\hline
\end{tabular}

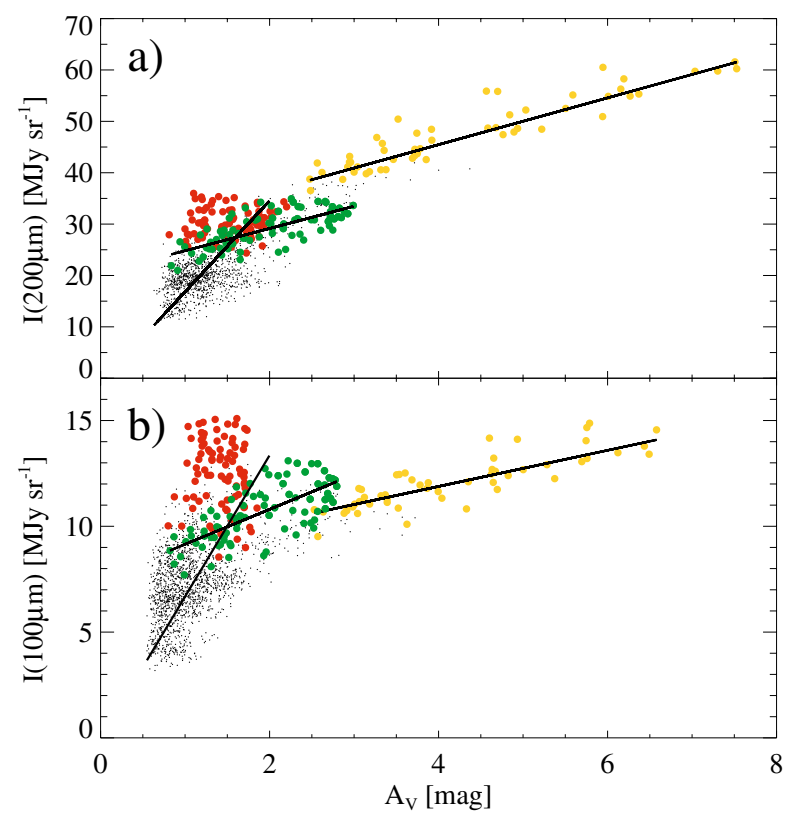

Fig. 2. a) Relation between visual extinction and $200 \mu \mathrm{m}$ surface brightness. The data points of the regions $\mathrm{A}, \mathrm{B}$ and $\mathrm{C}$ are marked with green, yellow, and red, respectively. Linear fits to regions $\mathrm{A} / \mathrm{B}$ and interclump part of the cloud are shown. We subtracted from $A_{\mathrm{V}}$ and $I(200 \mu \mathrm{m})$ the zero-level values as fixed outside the extent of L1642 (see Sect. 2.3.1). b) Relation between visual extinction and $100 \mu \mathrm{m}$ surface brightness. Linear fits to the regions $\mathrm{A} / \mathrm{B}$ and interclump part of the cloud are shown. The fit of the interclump part was forced to go through the origin. We subtracted from $A_{\mathrm{V}}$ and $I(100 \mu \mathrm{m})$ the zero-level values as fixed outside the extent of L1642 (see Sect. 2.3.1).

$A_{\mathrm{V}}$. We used the standard deviations of the zero-level values of each region as estimates of the last three error components.

\section{Results}

\subsection{Relation between visual extinction and far-IR surface brightness}

The relations between the $200 \mu \mathrm{m}$ and $100 \mu \mathrm{m}$ surface brightness and $A_{\mathrm{V}}$ are shown in Fig. 2. There is a clear difference between the overall relations; the $I(200 \mu \mathrm{m})$ vs. $A_{\mathrm{V}}$ relation shows less scatter, and the change of slope at $A_{\mathrm{V}} \approx 2-3$ mag is less pronounced than for $I(100 \mu \mathrm{m})$ vs. $A_{\mathrm{V}}$.

The parameters of linear fits to the data points $I(100 \mu \mathrm{m}) \mathrm{vs}$. $A_{\mathrm{V}}$ and $I(200 \mu \mathrm{m})$ vs. $A_{\mathrm{V}}$ of different regions are given in Table 1 in Cols. 5 and 6. For region $\mathrm{C}$, there is no clear correlation between far-IR intensity and $A_{\mathrm{V}}$, mainly because the $A_{\mathrm{V}}$-range in this region is rather small (see Fig. 1). It is notable that the

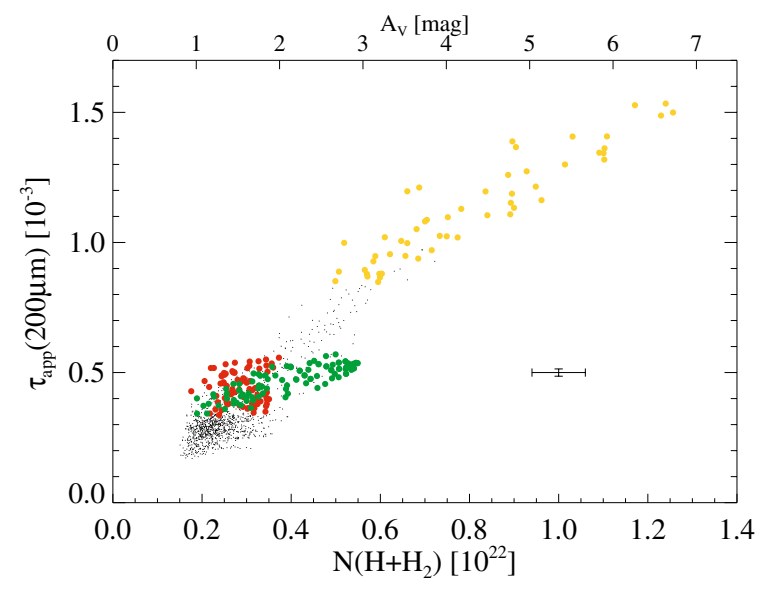

Fig. 3. Relation between the apparent $200 \mu \mathrm{m}$ optical depth and hydrogen column density $N\left(\mathrm{H}_{+} \mathrm{H}_{2}\right)$, derived from visual extinction (see Sect. 3.3). Typical pixel-to-pixel 1- $\sigma$ errors are shown. The data points of the regions $\mathrm{A}, \mathrm{B}$, and $\mathrm{C}$ are marked with green, yellow, and red, respectively. The values of $A_{\mathrm{V}}$ and $200 \mu \mathrm{m}$ optical depth have been derived after subtracting the zero-level values as fixed outside the extent of L1642 (see Sect. 2.3.1) from $A_{\mathrm{V}}$ and intensities $I(100 \mu \mathrm{m})$ and $I(200 \mu \mathrm{m})$.

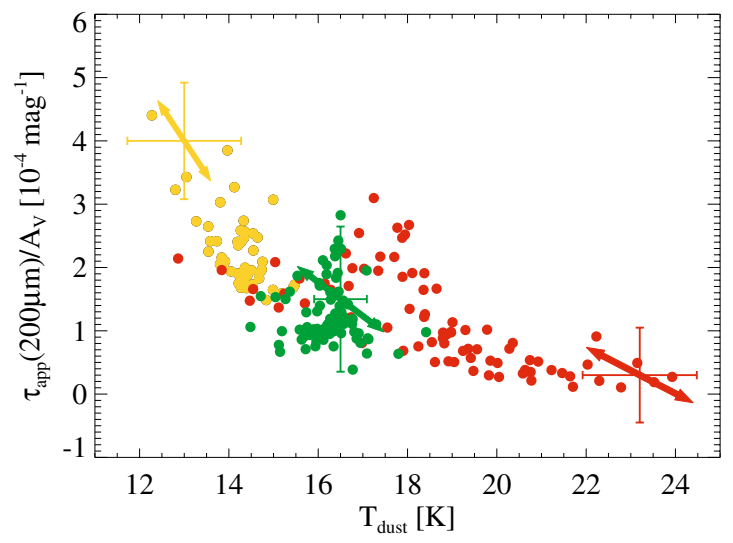

Fig. 4. The ratio $\tau_{\text {app }}(200 \mu \mathrm{m}) / A_{\mathrm{V}}$ as a function of dust temperature for the regions $\mathrm{A}, \mathrm{B}$, and $\mathrm{C}$ (green, yellow, and red, respectively), as derived with the method A in Sect. 3.2. The error bars show typical pixel-topixel errors within each region. The vectors show typical direction and magnitude (1- $\sigma$ standard deviation) of the movement of a data point within each region due to the inverse relation between $T_{\text {dust }}$ and $\tau$ (farIR), based on Monte Carlo calculations (see Sect. 4.2).

highest values of $I(100 \mu \mathrm{m})$ are located in region $\mathrm{C}$ where the visual extinction, $A_{\mathrm{V}} \approx 1.5 \mathrm{mag}$, is lower than in regions $\mathrm{A}$ and $\mathrm{B}$ (see Fig. 2b). This is due to the higher temperature in region $\mathrm{C}$ (see Figs. 3 and 4 of Paper I).

The general distribution of the data points is more linear at $200 \mu \mathrm{m}$ than at $100 \mu \mathrm{m}$. This can be explained by the fact that the temperature drop in region $\mathrm{B}$ influences the $100 \mu \mathrm{m}$ intensity more strongly than the $200 \mu \mathrm{m}$ intensity because the $100 \mu \mathrm{m}$ intensity is on the short- $\lambda$ side of the emission maximum.

\subsection{Determination of apparent emissivity $\tau_{\text {app }}(200 \mu m) / A_{V}$}

When investigating the possible variations in the dust emissivity in L1642, it is important to recognise that the optical depth as derived from the far-infrared observations at $100 \mu \mathrm{m}$ and $200 \mu \mathrm{m}$, i.e.

$\tau(200 \mu \mathrm{m})=I_{v}(200 \mu \mathrm{m}) / B_{v}\left(T_{\text {dust }}\right)$, 
is in general not the true optical depth of dust integrated over the line of sight. We designate the two quantities by apparent optical depth, $\tau_{\text {app }}(200 \mu \mathrm{m})$, and true optical depth, $\tau_{\text {true }}(200 \mu \mathrm{m})$ (see Sect. 4.3 for a detailed discussion).

The relation between $\tau_{\text {app }}(200 \mu \mathrm{m})$ and $A_{\mathrm{V}}$ is shown in Fig. 3. At $100 \mu \mathrm{m}$ the relation is exactly the same, except that the $\tau_{\text {app }}(100 \mu \mathrm{m})$ values are higher by a factor of $(200 / 100)^{2}=4$. The Pearson correlation coefficients for the relation are 0.85 and 0.91 for the regions $\mathrm{A}$ and $\mathrm{B}$, respectively.

We have determined the apparent emissivities using two different methods.

Method A. In this method we first subtract the zero level values corresponding to each region from the $100 \mu \mathrm{m}$ and $200 \mu \mathrm{m}$ intensities and visual extinction (see Sect. 2.3). Then the temperature and $200 \mu \mathrm{m}$ optical depth are derived on a pixel-by-pixel basis by using Eq. (1). The derived values of $\tau_{\text {app }}(200 \mu \mathrm{m})$ within different regions are given in Col. 8 of Table 1. Within the different regions, we derived the following mean values of apparent emissivity:

region $\mathrm{A}: 1.3 \pm 0.5 \times 10^{-4} \mathrm{mag}^{-1}$

region $\mathrm{B}: 2.2 \pm 0.6 \times 10^{-4} \mathrm{mag}^{-1}$

region $\mathrm{C}: 1.1 \pm 0.8 \times 10^{-4} \mathrm{mag}^{-1}$

interclump dust $\left(A_{\mathrm{V}}>1.2 \mathrm{mag}\right): 2.4 \pm 0.5 \times 10^{-4} \mathrm{mag}^{-1}$.

The $A_{\mathrm{V}}$ values of interclump dust have been limited to values greater than $1.2 \mathrm{mag}$ in order to suppress noise. The given error of emissivity is the 1- $\sigma$ standard deviation of individual datapoints within each region. The values of emissivity at $100 \mu \mathrm{m}$ are a factor of four $\left((200 / 100)^{2}\right)$ higher than the values at $200 \mu \mathrm{m}$.

Method B. From the slope of the $I(200 \mu \mathrm{m})$ vs. $I(100 \mu \mathrm{m})$ relation, we derive the dust temperature $T_{\text {dust }}$, not affected by any constant foreground and/or background emission components. This temperature represents a typical temperature in each region and is given in Col. 7 of Table 1 . Then, the $I(200 \mu \mathrm{m})$ vs. $A_{\mathrm{V}}$ relation (Fig. 2a) gives the slope between these quantities, called $k$. The apparent emissivity $\epsilon_{\text {app }}$ is then $\epsilon_{\text {app }}=k / B\left(T_{\text {dust }}\right)$. This method was applied to regions A and B and to interclump dust for which we can make a reliable linear fit of $I(200 \mu \mathrm{m})$ vs. $A_{\mathrm{V}}$. We derived the following mean values of apparent emissivity:

region $\mathrm{A}: 0.8 \pm 0.1 \times 10^{-4} \mathrm{mag}^{-1}$

region $\mathrm{B}: 1.9 \pm 0.3 \times 10^{-4} \mathrm{mag}^{-1}$

interclump dust: $2.2 \pm 0.2 \times 10^{-4} \mathrm{mag}^{-1}$.

These values are about a factor of 0.6-0.9 lower than those derived with method A above. More important, both of these independent methods give the same relative difference between regions $\mathrm{A}, \mathrm{B}$, and interclump dust.

\subsection{Apparent absorption cross section per $\mathrm{H}$-atom $\sigma_{\text {app }}^{H}(\lambda)$}

In this section we derive the ratio between the far-IR optical depth $\tau_{\text {app }}(\lambda)$ and the hydrogen column density, i.e. the apparent average absorption cross section per H-nucleus,

$\sigma_{\text {app }}^{\mathrm{H}}(\lambda)=\frac{\tau_{\text {app }}(\lambda)}{N(\mathrm{H})}$.

We can use the near-IR extinction to estimate the total hydrogen column density, $N\left(\mathrm{H}+\mathrm{H}_{2}\right)$. We adopt the value $N\left(\mathrm{H}+\mathrm{H}_{2}\right) / E(B-V)=5.8 \times 10^{21} \mathrm{~cm}^{-2} \mathrm{mag}^{-1}$ valid for diffuse clouds (Bohlin et al. 1978), together with $A_{\mathrm{V}} / E(B-V)=3.1$,

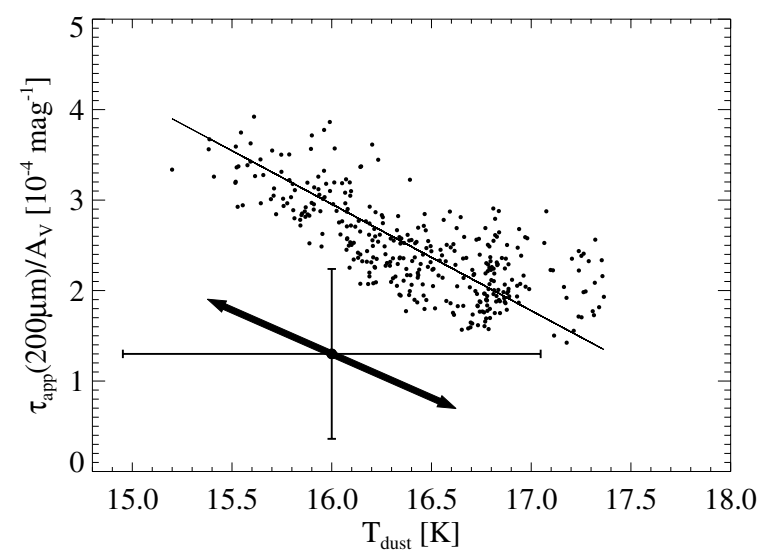

Fig. 5. The ratio $\tau_{\text {app }}(200 \mu \mathrm{m}) / A_{\mathrm{V}}$ as a function of dust temperature for the interclump part of the cloud, as derived with method A in Sect. 3.2. To suppress noise, we have included only those datapoints for which $A_{\mathrm{V}}>1.2 \mathrm{mag}$. The error bars show typical pixel-to-pixel errors. The straight line has a slope of $-1.2 \times 10^{-4}$. The vector shows typical direction and magnitude (1- $\sigma$ standard deviation) of the movement of a data point due to the inverse relation between $T_{\text {dust }}$ and $\tau$ (far-IR), based on Monte Carlo calculations (see Sect. 4.2).

to obtain $N\left(\mathrm{H}+\mathrm{H}_{2}\right) / A_{\mathrm{V}}=1.87 \times 10^{21} \mathrm{~cm}^{-2} \mathrm{mag}^{-1}$. The relation between $N\left(\mathrm{H}+\mathrm{H}_{2}\right)$ and $\tau_{\text {app }}(200 \mu \mathrm{m})$ is shown in Fig. 3. Within the different regions, we derived the following mean values of $\sigma_{\mathrm{app}}^{\mathrm{H}}(200 \mu \mathrm{m})$, calculated with the method A above (see Sect. 3.2):

region A: $0.7 \pm 0.3 \times 10^{-25} \mathrm{~cm}^{2} \mathrm{H}$-atom ${ }^{-1}$ region $\mathrm{B}$ : $1.2 \pm 0.5 \times 10^{-25} \mathrm{~cm}^{2} \mathrm{H}$-atom ${ }^{-1}$ region $\mathrm{C}$ : $0.6 \pm 0.4 \times 10^{-25} \mathrm{~cm}^{2} \mathrm{H}$-atom ${ }^{-1}$ interclump dust: $1.3 \pm 0.3 \times 10^{-25} \mathrm{~cm}^{2} \mathrm{H}$-atom ${ }^{-1}$.

The given error of $\sigma_{\text {app }}^{\mathrm{H}}(\lambda)$ is $1-\sigma$ standard deviation of individual datapoints within each region. The values of $\sigma_{\text {app }}^{\mathrm{H}}(100 \mu \mathrm{m})$ are four times as high as the values of $\sigma_{\text {app }}^{\mathrm{H}}(200 \mu \mathrm{m})$.

\section{4. $\tau_{\text {app }}(200 \mu \mathrm{m}) / A_{V}$ as a function of $T_{\text {dust }}$}

Figure 4 shows the emissivity $\tau_{\mathrm{app}}(200 \mu \mathrm{m}) / A_{\mathrm{V}}$ as a function of dust temperature for the regions $\mathrm{A} / \mathrm{B} / \mathrm{C}$, as derived with method A (see Sect. 3.2). The emissivity appears to increase as the temperature decreases. Taken from Table 2, there is two-fold increase in emissivity in the temperature range $\sim 19-14 \mathrm{~K}$, the range determined by the mean temperatures of regions $\mathrm{C}$ and $\mathrm{B}$. At the very lowest temperatures, $12-14 \mathrm{~K}$, there is indication of a further increase of emissivity, but the number of data points is not sufficient to confirm that.

The emissivity as a function of dust temperature for the interclump dust is shown in Fig. 5. The emissivity appears to increase by a factor of about 1.5 when the dust temperature decreases from $17 \mathrm{~K}$ to $15.5 \mathrm{~K}$.

In order to confirm the validity of the relation between emissivity and dust temperature, we must exclude the possibity that the relation is due to uncertainties in the derived dust temperature: because the optical depth depends inversely on dust temperature $\left(\tau \propto I / B_{\text {dust }}\right)$, the $x$ - and $y$-axes in Figs. 4 and 5 are not independent. Any overestimation of temperature causes underestimation of optical depth and vice versa, and as a consequence a relation like that in Figs. 4 or 5 may occur. We estimated the uncertainty of the emissivity via Monte Carlo calculations, considering the eight error sources as listed in Table 2. For details see 
Appendix C. We conclude that the increase in emissivity with decreasing $T_{\text {dust }}$ within region B (yellow points in Fig. 4) can be partly caused by errors in the $T_{\text {dust }}$ estimates. However, the increased emissivity in region $\mathrm{B}$ compared to regions $\mathrm{A}$ and $\mathrm{C}$ cannot be explained this way. It is found to be a real effect caused by the change in dust properties. On the other hand, the range of temperature in Fig. 5 is rather small in the case of interclump dust, and thus the change of emissivity is only slightly larger than the derived uncertainty.

\section{Discussion}

\subsection{Dust emissivity variations}

The relatively large area of $1.6 \square^{\circ}$, which we mapped in and around L1642, contains both dense and more diffuse areas with different dust populations. The $200 \mu \mathrm{m}$ and $100 \mu \mathrm{m}$ emission, dominated by the large particles, and the $60 \mu \mathrm{m}, 25 \mu \mathrm{m}$, and $12 \mu \mathrm{m}$ emissions, dominated by smaller particles, peak at separate positions in the cloud (see e.g. Laureijs et al. 1987). Compared with the situation where a sample of distinct clouds have been studied to cover a range of different dust properties (see e.g. del Burgo et al. 2003), we have the observational advantage that a more accurate (relative) calibration across our single, uniformly mapped area can be achieved. Thus, the variations of the dust properties over the mapped area are more accurately determined.

We found in Sect. 3.4 that the emissivity of the dust, $\tau_{\text {app }}(200 \mu \mathrm{m}) / A_{\mathrm{V}}$, is not constant but decreases as a function of dust temperature, $T_{\text {dust }}$. Part of this variation is caused by the $x$ - and $y$-axes not being independent. Our error analysis in Appendix $C$ has shown, however, that these tendencies are real on the large scale over the whole cloud area.

Based only on the temperature, the emissivity of interclump dust $\left(T_{\text {dust }} \approx 16.3-17.1 \mathrm{~K}\right)$ is expected to be at about the same level as the emissivity of region $\mathrm{A}\left(T_{\text {dust }} \approx 15.9-16.3 \mathrm{~K}\right)$. However, it is about two times higher, at the same level as the emissivity in region B. This can be explained in terms of different contributions by large grains in different regions of L1642. The maps of Laureijs et al. (1987) show that $25 \mu \mathrm{m}$ emission has local maxima at regions $\mathrm{A}$ and $\mathrm{C}$, while $60 \mu \mathrm{m}$ emission has a local maximum at region $\mathrm{C}$. This indicates that very small grains (VSGs) have a larger abundance in regions $\mathrm{A}$ and $\mathrm{C}$ as compared to other regions. One possible explanation for the lower far-IR emissivity of big grains as observed in these regions $\mathrm{A}$ and $\mathrm{C}$ could be the following: the big grains and VSGs are part of the same grain size distribution; decrease in the number of VSGs means an increase in the number of big grains, e.g. via coagulation or mantle formation.

There is, in addition, the question whether the $100 \mu \mathrm{m}$ emission in regions $\mathrm{A}$ and $\mathrm{C}$ contains a contribution from transiently heated very small grains, which would make the derived temperature an ill-defined quantity. This is discussed in Appendix D, and the conclusion is that there is no evidence of substantial emission at $100 \mu \mathrm{m}$ from transiently heated VSGs in regions A or $\mathrm{C}$.

\subsection{Dust emissivity versus temperature: modelling}

In Paper I we showed that the decrease in dust temperature towards the centre of region B in L1642 cannot be explained solely by the attenuation of the radiation field. In the denser parts of a cloud, the dust grains are expected to stick together, forming grains with a bigger cross-section. We used the radiative transfer program of Juvela \& Padoan (2003) to test if an increased value of the absorption cross-section (that is, increased emissivity) of dust grains can explain the observed decrease in dust temperature in L1642.

The parameters of the model cloud that are based on observations are the cloud size, radial density distribution, and visual extinction $A_{\mathrm{V}}$ at the cloud centre. Our model is tailored for region B in L1642. The $F W H M$ size of $\tau(200 \mu \mathrm{m})$ of the model is equal to the observed one. The density is constant up to 0.2 times the cloud radius, outside of which it has the form $\rho(r) \propto r^{-1.5}$. This density distribution agrees with the $\mathrm{CO}$ observations (Russeil et al. 2002) and the far-IR observations (Paper I). The cloud is heated by the Solar neighbourhood interstellar radiation field (Mathis et al. 1983). The grain properties used in the model are those of Li \& Draine (2001b). We calculated a set of five models, multiplying the values of absorption cross-sections given by $\mathrm{Li}$ $\&$ Draine by a factor of $k=1.0,1.5,2.0,3.0,4.0$, and 5.0, at all wavelengths longer than an arbitrarily chosen threshold wavelength of $50 \mu \mathrm{m}$.

The starting point of the model is the observed maximum visual extinction of $A_{\mathrm{V}}=6.7 \mathrm{mag}$ at $4.5^{\prime}$ resolution. First, in the case of $k=1.0$, the column density of dust is modified until the model gives a value of $A_{\mathrm{V}}$ that is equal to the observed one; $A_{\mathrm{V}}$ (model) is derived by integrating the extinction coefficient along the line of sight through the model cloud. Then, in subsequent calculations, with increasing values of $k$, the true column density of dust in the model is kept the same as in the case $k=1.0$. The value of visual extinction given by the model is the same for all values of $k$ because column density of dust is constant and the properties of dust particles are changed only at $\lambda>50 \mu \mathrm{m}$.

The model gives the $100 \mu \mathrm{m}$ and $200 \mu \mathrm{m}$ intensities at different radial distances from the cloud centre. The intensities are convolved to $4.5^{\prime}$ resolution. The optical depth derived from $100 \mu \mathrm{m}$ and $200 \mu \mathrm{m}$ intensities given by the model cloud is an apparent optical depth, because it is obtained by mimiking the actual ISO observations. The true optical depth, on the other hand, is derived by integrating the absorption coefficient along the line of sight through the model cloud. We then derive maps of colour temperature and apparent optical depth $\tau_{\text {app }}(200 \mu \mathrm{m})$. The values of temperature and emissivity, $\tau_{\mathrm{app}}(200 \mu \mathrm{m}) / A_{\mathrm{V}}$, at the centre of the model cloud are shown in Fig. 6 for different values of $k$, together with the observed values of temperature and emissivity. The $1-\sigma$ uncertainties of the observed values were derived by assuming $20 \%$ absolute uncertainty for the $100 \mu \mathrm{m}$ and $200 \mu \mathrm{m}$ intensities. Clearly, values of absorption cross-sections at far-IR enhanced by a factor of about $2-3$ are required to explain both the observed temperature and apparent emissivity towards the centre of the cloud.

In the case $k=2$, the model predicts intensities at the cloud centre of about $15 \mathrm{MJy} \mathrm{sr}^{-1}$ and $64 \mathrm{MJy} \mathrm{sr}^{-1}$ at $100 \mu \mathrm{m}$ and $200 \mu \mathrm{m}$, respectively, at $4.5^{\prime}$ resolution. These values compare well with the observed ones, $\sim 14 \mathrm{MJy} \mathrm{sr}^{-1}$ and $\sim 60 \mathrm{MJy} \mathrm{sr}^{-1}$.

\subsection{The difference between true and apparent emissivity}

As discussed in the previous section, the apparent value of emissivity is always lower than its true value. The underlying reason is that the colour temperature is biased towards values that are too high, leading to an apparent optical depth that is too small. This bias is caused by the following facts: i) there is a temperature gradient towards the cloud centre with lower temperatures at the centre than at the edge (see e.g. Zucconi et al. 2001 and 


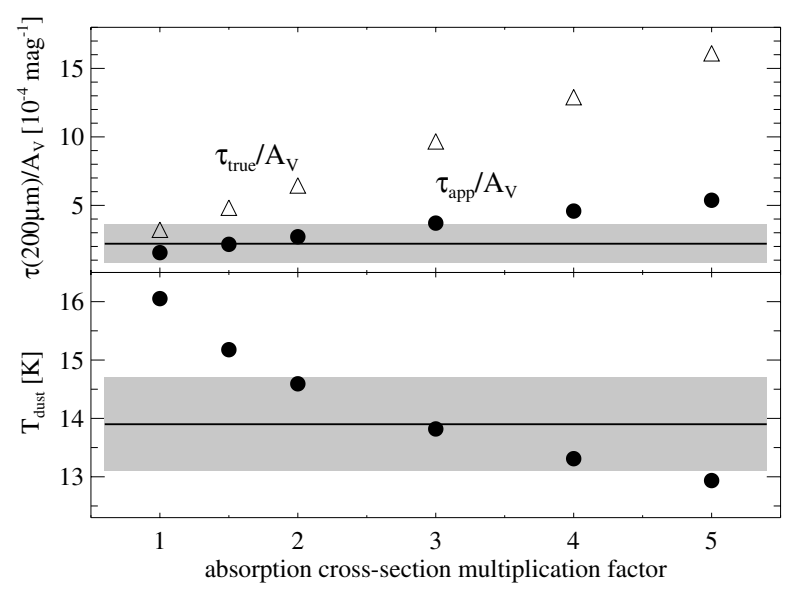

Fig. 6. Model clouds: Apparent dust temperature and emissivity $\left(\tau_{\text {app }}(200 \mu \mathrm{m}) / A_{\mathrm{V}}\right)$ as a function of multiplication factor $k$ for the absorption cross-section of dust grains at far-IR (see Sect. 4.2 for details). The dots are values of apparent temperature and emissivity as derived from the $100 \mu \mathrm{m}$ and $200 \mu \mathrm{m}$ intensities given by the model. The observed mean values of temperature and emissivity in the centre of region $\mathrm{B}$ are marked as horizontal lines, and shaded areas show the 1- $\sigma$ uncertainties. The triangles are the true values of emissivity.

Galli et al. 2002) for a theoretical discussion); ii) graphite particles are warmer than silicate particles, which furthermore extends the temperature range of dust particles; iii) the very small grains, which have a larger temperature fluctuation range than the big grains, make a noticeable contribution at $100 \mu \mathrm{m}$. The net result of the above effects is that the emission is to some degree dominated by the warmer dust particles; they contribute a larger fraction of the total emission than what should be accounted for by their relative mass. This is especially true at $100 \mu \mathrm{m}$. The colour temperature derived from $100 \mu \mathrm{m}$ and $200 \mu \mathrm{m}$ observations is therefore larger than the proper, mass-weighted temperature. Thus, the apparent optical depth $\tau_{\text {app }}(200 \mu \mathrm{m})$ is always smaller than the true optical depth $\tau_{\text {true }}(200 \mu \mathrm{m})$. Consequently, the apparent emissivity is always smaller than the true emissivity.

Irrespective of the dust temperature, an increase of absorption cross-section of dust particles produces an increase of optical depth by the same amount. In Fig. 6, the apparent emissivity is seen to increase only by a factor of about 3.5 when $k$ increases from one to five. This is due to the fact that the above mentioned effects are more pronounced when the temperature is lower.

\subsection{Comparison with grain models and other clouds}

The observed two-fold increase in emissivity in L1642 is similar to that observed in other regions; increase at $850 \mu \mathrm{m}$ by a factor of $\sim 4$ over a temperature range $\sim 20 \mathrm{~K}-12 \mathrm{~K}$ in the dark cloud IC5146 (Kramer et al. 2003) and by a factor of $\sim 2$ in Barnard 68 (Bianchi et al. 2003); increase in the FIR by a factor of $~ 3.4$ in a dense filament in Taurus (Stepnik et al. 2003); and increase by a factor of $>4$ in high-latitude clouds (del Burgo et al. 2003).

Table 1 gives a compilation of some observed and theoretical properties of dust. The theoretical values for $\tau(200 \mu \mathrm{m}) / A_{\mathrm{V}}$ and $\sigma^{\mathrm{H}}(200 \mu \mathrm{m})$ in Table 1 are "true" values in our nomenclature. Because the "apparent" values of $\sigma^{\mathrm{H}}($ far-IR) and emissivity are always lower than the true values (see Sect. 4.3), a comparison with the model results is not straightforward. In fact, one should use a certain model to derive intensity values at the two far-IR wavelengths, $100 \mu \mathrm{m}$, and $200 \mu \mathrm{m}$, and use them to derive the apparent optical depth and emissivity, as we have done in Sect. 4.2.

The value of apparent $\sigma_{\text {app }}^{\mathrm{H}}(200 \mu \mathrm{m})$ in region B is slightly lower than the true value obtained for diffuse ISM by Li \& Draine (2001a) for their carbonaceous-silicate grain model in the case $R_{\mathrm{V}}=3.1$. By using Fig. 6 we can transform the value of $\sigma_{\mathrm{app}}^{\mathrm{H}}(200 \mu \mathrm{m})$ of region B into its true value. By assuming that the multiplication factor for emissivity $k=2.5$, the ratio of true emissivity to apparent emissivity for the model is about 2.5. The value of $\sigma_{\text {true }}^{\mathrm{H}}(200 \mu \mathrm{m})$ in region B would then be about $3.0 \times 10^{-25}$. This is higher than any value for diffuse ISM in Table 1.

The regions $\mathrm{A}$ and $\mathrm{C}$ have $\left\langle A_{\mathrm{V}}\right\rangle=1.5-2.1 \mathrm{mag}$, slightly higher than the extinction through typical diffuse dust. In these regions the value of apparent $\sigma_{\text {app }}^{\mathrm{H}}(200 \mu \mathrm{m})$ (as measured with method A) is clearly less than the true value obtained for diffuse ISM by Li \& Draine. However, we cannot compare the estimated values of true $\sigma_{\text {true }}^{\mathrm{H}}(200 \mu \mathrm{m})$ of regions $\mathrm{A}$ and $\mathrm{C}$ to the true value of the model by Li \& Draine because we lack radiative transfer calculations for regions $\mathrm{A}$ and $\mathrm{C}$.

Mantle accretion on dust grains can increase emissivity by a factor of about two at most (Preibisch et al. 1993), while grain-grain coagulation (Bazell \& Dwek 1990) can increase the emissivity by a larger amount. Thus, either of these models can explain the observed increase in emissivity by a factor of two between regions $\mathrm{B}$ and $\mathrm{C}$.

\section{Conclusions}

Based on a large-scale $200 \mu \mathrm{m}$ ISOPHOT mapping of the cloud Lynds 1642, combined with $100 \mu \mathrm{m}$ IRAS/ISSA map and an extinction map, we draw the following main conclusions.

- We have found that one has to make a distinction between apparent values of parameters, such as temperature, optical depth, and emissivity, and their true values. The apparent values are those derived from the intensity maps at $100 \mu \mathrm{m}$ and $200 \mu \mathrm{m}$. We find that the apparent optical depth and emissivity are always smaller than their true values because the apparent temperature is biased towards higher values.

- The apparent emissivity $\tau_{\text {app }}\left(\right.$ far-IR)/ $/ A_{\mathrm{V}}$ increases with decreasing dust temperature. There is about a two-fold increase in apparent emissivity when dust temperature decreases from $19 \mathrm{~K}$ to $14 \mathrm{~K}$.

- Radiative transfer modelling shows that an increase in the absorption cross section of dust at far-IR is capable of explaining two phenomena observed in L1642: i) a decrease in dust temperature towards the centre of the dense region $B$ by an amount that is more than what can be explained solely by the attenuation of interstellar radiation field; ii) an increase in apparent emissivity towards the colder regions. We find that an increase in the far-IR cross sections by a factor of about $2-3$ is required to explain both the temperature drop and the observed emissivity increase in region B.

Acknowledgements. The work of K.L., M.J., and K.M. has been supported by the Finnish Academy through grants Nos. 1204415, 1210518, and 1201269, which is gratefully acknowledged. This publication makes use of data products from the Two Micron All Sky Survey, which is a joint project of the University of Massachusetts and the Infrared Processing and Analysis Center/California Institute of Technology, funded by the National Aeronautics and Space Administration and the National Science Foundation. This research made use of the USNOFS Image and Catalogue Archive operated by the United States Naval Observatory, Flagstaff Station (http://www.nofs.navy.mil/data/fchpix). 


\section{References}

Adams, F. C. 1991, ApJ, 382, 544

Bazell, D., \& Dwek, E. 1990, ApJ, 360, 142

Bianchi, S., Gonçalves, J., Albrecht, M., et al. 2003, A\&A, 399, L43

Bohlin, R. C., Savage, B. D., \& Drake, J. F. 1978, ApJ, 224, 132

del Burgo, C., \& Laureijs, R. J. 2005, MNRAS, 360, 901

del Burgo, C., Laureijs, R. J., Ábrahám, P., \& Kiss, Cs. 2003, MNRAS, 346, 403

Cambrésy, L., Boulanger, F., Lagache, G., \& Stepnik, B. 2001, A\&A, 375, 999

Cambrésy, L., Jarrett, T. H., \& Beichman, C. A. 2005 [arXiv: astro-ph/0501444]

Casali, M. M. 1986, MNRAS, 223, 341

Désert, F.-X., Boulanger, F., \& Puget, J. L. 1990, A\&A, 237, 215

Dwek, E. 1997, ApJ, 484, 779

Dwek, E., Arendt, R. G., Fixsen, D. J., et al. 1997, ApJ, 475, 565

Evans, D. W. 1989, A\&AS, 78, 249

Gabriel, C., Acosta-Pulido, J., Heinrichsen, I., et al. 1997, in Proc. of the ADASS

VI conference, ed. G. Hunt, \& H. E. Payne, ASP Conf. Ser., 125, 108

Galli, D., Walmsley, C. M., \& Gonçalves, J. 2002, A\&A, 394, 275

Hearty, T., Fernández, M., Alcalá, J. M., et al. 2000, A\&A, 357, 681

Hildebrand, R. H. 1983, QJRAS, 24, 267

Juvela, M., \& Padoan, P. 2003, A\&A, 397, 201

Juvela, M., Mattila, K., Lehtinen, K., et al. 2002, A\&A, 382, 583

Kramer, C., Richer, J., Mookerjea, B., et al. 2003, A\&A, 399, 1073

Kessler, M. F., Steinz, J. A., \& Anderegg, M. E. 1996, A\&A, 315, L27

Laureijs, R. J., Mattila, K., \& Schnur, G. 1987, A\&A, 184, 269

Laureijs, R. J., Haikala, L., Burgdorf, M., et al. 1996, A\&A, 315, L317

Laureijs, R. J., Herbstmeier, U., Ábrahám, P., Klaas, U., \& Lemke, D. 2000, in ISO Beyond Point Sources: Studies of Extended Infrared Emission, ed. R. J. Laureijs, K. Leech, \& M. F. Kessler, ESA SP-455, 131
Lehtinen, K., Lemke, D., Mattila, K., \& Haikala, L. K. 1998, A\&A, 333, 702 Lehtinen, K., Russeil, D., Juvela, K., Mattila, K., \& Lemke, D. 2004, A\&A, 423, 975, Paper I

Lemke, D., Klaas, U., Abolins, J., et al. 1996, A\&A, 315, L64

Li, A., \& Draine, B. T. 2001a,

http://www .astro.princeton.edu/ draine/dust/dustmix

Li, A., \& Draine, B. T. 2001b, ApJ, 554, 778

Lombardi, M., \& Alves, J. 2001, A\&A, 377, 1023

Mathis, J. S. 1990, ARA\&A, 28, 37

Mathis, J. S., Mezger, P. G., \& Panagia, N. 1983, A\&A, 128, 212

Mezger, P. G., Mathis, J. S., \& Panagia, N. 1982, A\&A, 105, 372

Monet, D. G., Levine, S. E., Canzian, B., et al. 2003, AJ, 125, 984

Ossenkopf, V., \& Henning, T. 1994, A\&A, 291, 943

Preibisch, T., Ossenkopf, V., Yorke, H. W., et al. 1993, A\&A, 279, 577

Rawlings, M. G., Juvela, M., Mattila, K., Lehtinen, K., \& Lemke, D. 2004, MNRAS, 356, 810

Reipurth, B., \& Heathcote, S. 1990, A\&A, 229, 527

Ridderstad, M., Juvela, M., Lehtinen, K., Lemke, D., \& Liljeström, T. 2006, A\&A, 451, 961

Russeil, D., Juvela, M., Lehtinen, K., Mattila, K., \& Paatero, P. 2003, A\&A, 409, 135

Sandell, G., Reipurth, B., \& Gahm, G. 1987, A\&A, 181, 283

Schlegel, D. J., Finkbeiner, D. P., \& Davis, M. 1998, AJ, 500, 525

Stepnik, B., Abergel, A., Bernard, J.-P., et al. 2003, A\&A, 398, 551

Wolf, M. 1923, Astron. Nachr., 219, 109

Yun, J. L., \& Clemens, D. P. 1991, ApJ, 381, 474

Zucconi, A., Walmsley, C. M., \& Galli, D. 2001, A\&A, 376, 650 
K. Lehtinen et al.: ISO far-infrared observations of the high-latitude cloud L1642. II., Online Material $p 1$

\section{Online Material}




\section{Appendix A: B- and I-band extinction from starcounts}

Data for star counting on $B$ - and $I$-bands have been extracted from the USNO-B1.0 archive (Monet et al. 2003). We used data from the second epoch SERC- $J$ ( $B_{J}$-band) and SERC- $I$ ( $I$-band) surveys. For the effective wavelengths of the $B$ and $I$ bands, we used $470 \mathrm{~nm}$ and $810 \mathrm{~nm}$, respectively (see Evans 1989).

We were forced to use a reference area relatively close to the $\mathrm{L} 1642$ (at RA $(\mathrm{J} 2000)=4^{\mathrm{h}} 35^{\mathrm{m}} 00^{\mathrm{s}}$, Dec. $(\mathrm{J} 2000)=-16^{\circ} 00^{\prime} 00^{\prime \prime}$, $l=212.90^{\circ}, b=-37.26^{\circ}$, a circular region of $1^{\circ}$ radius), in order to have the reference stars on the same plate as most of the field stars and, thus, with the same magnitude calibration. The value of $E(B-V)$ at the reference position is about $0.12 \mathrm{mag}$ according to the dust map of Schlegel et al. (1998), which corresponds to $A_{\mathrm{V}} \approx 0.4 \mathrm{mag}$. Star counting was performed in circles with a radius of $3^{\prime}$. Extinctions were derived from the $\log N(m)$ vs. $m$ curves in the cloud and reference areas using the method of Wolf (1923). The pixel size of the extinction map was chosen to be $3^{\prime}$.

Figure A.1 shows the $B$-band extinction map, after the original map was smoothed with a Gaussian with $F W H M=4.5^{\prime}$. The maximum extinction on the smoothed map, $A(B) \approx 3.0 \mathrm{mag}$, occurs close to the center of region $\mathrm{B}$. In the center of region $\mathrm{C}$ the extinction is about $1.8 \mathrm{mag}$, while in regions $\mathrm{A} 1$ and $\mathrm{A} 2$ the extinctions are about $0.8 \mathrm{mag}$ and $1.0 \mathrm{mag}$, respectively.

The relation between the $B$ - and $I$-band extinctions is shown in Fig. A.2. The extinction ratios at $B$ - and $I$-bands $(470 \mathrm{~nm}$ and $810 \mathrm{~nm}$ ) for the cases $R_{\mathrm{V}}=3.1$ (diffuse dust) and $R_{\mathrm{V}}=5.0$ ("outer-cloud" dust) were adopted from Table 1 of Mathis (1990) and are shown as dashed lines. These values of $R_{\mathrm{V}}$ correspond to slopes of 0.48 and 0.58 in Fig. A.2. The two types of linear fits to the data points, solid and dotted lines, give slopes of $0.54 \pm 0.02$ and $0.49 \pm 0.02$, respectively. We may thus conclude that the extinction law in L1642 is similar to that of diffuse interstellar matter.

There is indication in Fig. A.2 that region $C$ has a lower value of $R_{\mathrm{V}}$ than region $\mathrm{B}$. In addition, at $A_{\mathrm{B}} \gtrsim 3$ mag the relation indicates a higher value for $R_{\mathrm{V}}$ inside region $\mathrm{B}$. The number and accuracy of data points is insufficient for firm conclusions, though.

\section{Appendix B: Extinction from near-IR colour excesses}

As reference fields, representing areas with "no extinction", we selected two 40' diameter circular areas located closely at the same galactic latitude as L1642, at $\mathrm{RA}(\mathrm{J} 2000)=4^{\mathrm{h}} 32^{\mathrm{m}} 6^{\mathrm{s}}$, $\operatorname{Dec}(\mathrm{J} 2000)=-12^{\circ} 50^{\prime} 27^{\prime \prime}$, and $\mathrm{RA}(\mathrm{J} 2000)=4^{\mathrm{h}} 41^{\mathrm{m}} 22^{\mathrm{s}}$, $\operatorname{Dec}(\mathrm{J} 2000)=-16^{\circ} 41^{\prime} 44^{\prime \prime}$.

The observed colours of stars in the reference areas have the mean values and standard deviations of $(J-H)=0.52 \pm 0.17$ and $(H-K)=0.19 \pm 0.19$. They will be used as estimates of the intrinsic colours $(J-H)_{0},(H-K)_{0}$, and their variances. For the ratio of visual extinction to colour excess we used $A_{\mathrm{V}} / E(J-H)=$ 8.86 and $A_{\mathrm{V}} / E\left(H-K_{\mathrm{s}}\right)=15.98$, which correspond to $R_{\mathrm{V}}=3.1$ (Mathis 1990).

The pixel size in the extinction map was chosen to be the same as the pixel size of ISO C200 detector, i.e. 1.5'. The extinction value for each map pixel was derived by using a Gaussian as a weighting function for the extinction values of individual stars and applying the sigma-clipping smoothing technique of Lombardi \& Alves (2001). The size of the Gaussian function depends on whether we compare visual extinction with $200 \mu \mathrm{m}$ ISO map or with maps from IRAS data. In the case of ISO map,

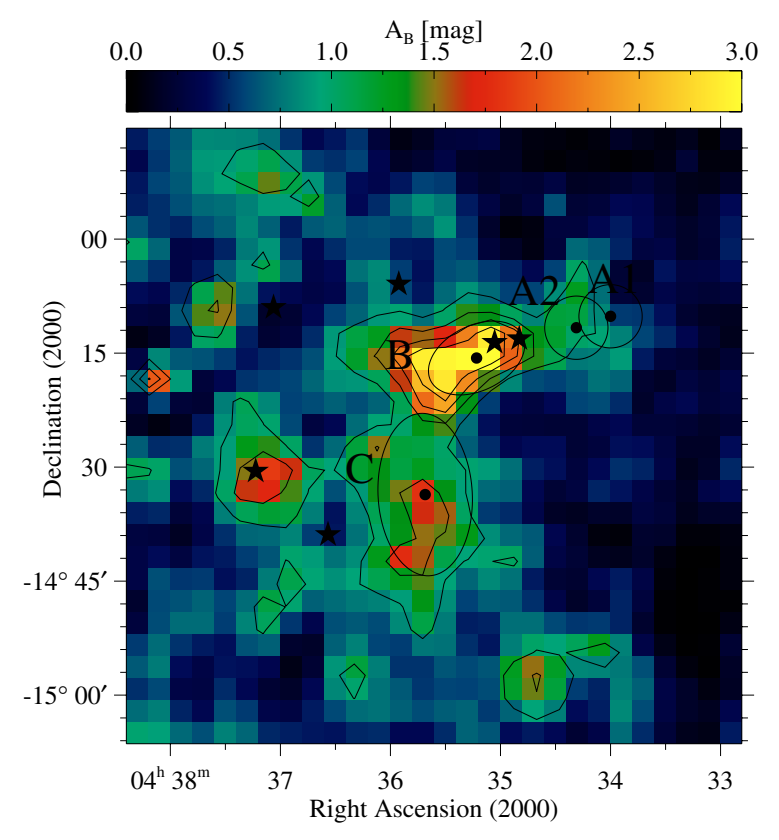

Fig. A.1. Extinction through L1642 in $B$-band. The IRAS sources, marked with asterisks, are from right to left: IRAS 04325-1419, 043271419, 04336-1412, 04342-1444, 04347-1415, and 04349-1436. The FWHM sizes of the regions A1, A2, B, and C as derived by Lehtinen et al. (Paper I) from $200 \mu \mathrm{m}$ optical depth data are shown as ellipses and circles. The contours are at 1.0, 1.5, 2.0, and 2.5 mag.

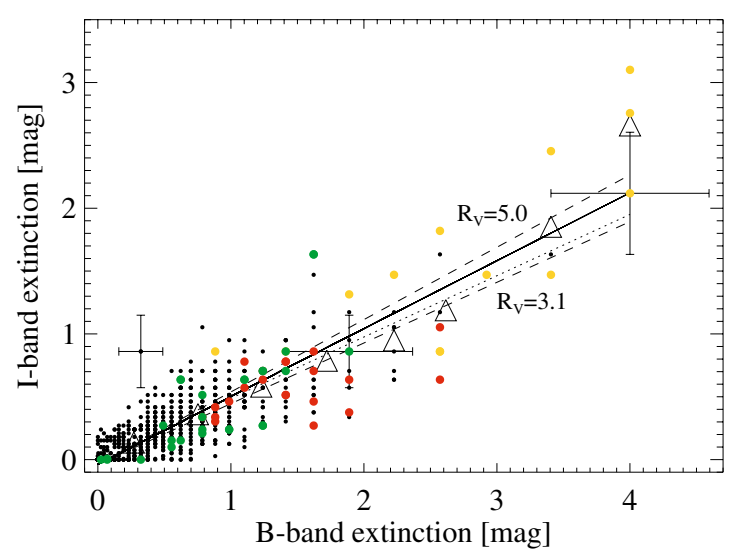

Fig. A.2. Relation between star count based $B$ - and $I$-band extinction. The data points of region A, B, and C are plotted in green, yellow, and red, respectively. The slopes of the dashed lines correspond to the ratios of total to selective extinction $R_{\mathrm{V}}=3.1$ and $R_{\mathrm{V}}=5.0$. The solid line is a linear fit for $A_{\mathrm{B}}<3.5 \mathrm{mag}$, weighting by the error of extinction. The triangles are mean values of extinctions in $0.5 \mathrm{mag} B$-band intervals up to $4.0 \mathrm{mag}$, and the dotted line is a linear fit of type $y=b x$ to these mean values for $A_{\mathrm{B}}<3.5 \mathrm{mag}$. Some examples of $1-\sigma$ errors are shown.

we used a Gaussian with $F W H M=3.5^{\prime}$, limited by the necessity of having a sufficient signal-to-noise ratio for the extinction. The point-spread profile of ISO at $200 \mu \mathrm{m}$ can be described by a Gaussian function having $F W H M=86^{\prime \prime}$. Thus, the ISO map was convolved with a Gaussian having $F W H M=$

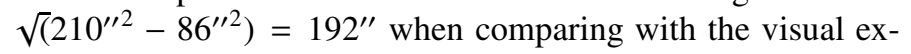
tinction map having $F W H M=3.5^{\prime}$ resolution.

The temperature and optical depth maps were made by utilizing IRAS data that have a $F W H M=4.5^{\prime}$ resolution, measured from intensity profiles of point sources on the IRAS map. For these maps the $200 \mu \mathrm{m}$ ISO data were convolved with a Gaussian 
having $\left.F W H M=\sqrt{\left(270^{\prime \prime 2}\right.}-86^{\prime \prime 2}\right)=256^{\prime \prime}$. Consequently, we use the visual extinction map with $F W H M=4.5^{\prime}$ resolution in the context of temperature and optical depth maps.

There are two sources of error for the extinction map; variance of the intrinsic colours $(J-H)_{0}$ and $(H-K)_{0}$ and variance of the observed magnitudes of the programme stars. In the $A_{\mathrm{V}}$ map made with a $F W H M=4.5^{\prime}$ Gaussian the former source dominates with a $\sim 0.29$ mag $1-\sigma$ error per pixel, while the latter source typically contributes $\sim 0.08 \mathrm{mag} 1-\sigma$ error per pixel, giving a typical total error of $\sim 0.32$ mag per pixel.

\section{Appendix C: Error analysis of the emissivity}

We estimated the uncertainty of the $200 \mu \mathrm{m}$ emissivity via Monte Carlo calculations, considering the eight error sources as listed in Table 2. For the emissivity estimation, the largest error source is the error of $A_{\mathrm{V}}$, because it is a large fraction of the extinction $\left(\sim 10-75 \%\right.$ for $\left.A_{\mathrm{V}}=6-1 \mathrm{mag}\right)$. On the other hand, the error of $A_{\mathrm{V}}$ does not cause any inverse emissivity vs. $T_{\text {dust }}$ relation. Typical errors of emissivity and temperature, based on Monte Carlo calculations, are plotted in Fig. 4 for each of the region $\mathrm{A} / \mathrm{B} / \mathrm{C}$ and for interclump dust in Fig. 5.

We used the Monte Carlo method to study which direction and how much a datapoint moves in a $T_{\text {dust }}$ vs. $\tau$ (far-IR) plot due to pixel-to-pixel errors in the $100 \mu \mathrm{m}$ and $200 \mu \mathrm{m}$ intensity maps. Let us first consider the non-realistic case where only one of the intensity maps has errors. In this case there is always a negative correlation between $T_{\text {dust }}$ and $\tau$ (far-IR), just as expected. In the case where both of the intensity maps have errors, positive correlation between $T_{\text {dust }}$ and $\tau$ (far-IR) is also possible. This happens when $100 \mu \mathrm{m}$ and $200 \mu \mathrm{m}$ intensities either increase or decrease simultaneously. For example, if both of them increase by a suitable amount, the derived temperature may increase just slightly, and then the optical depth increases too because the relative increase in intensity may be greater than the influence of the increase in temperature. However, a positive correlation between $T_{\text {dust }}$ and $\tau$ (far-IR) happens only when the change of temperature is minuscule. When taking all the errors of Table 2 into account, we find that the slope of the inverse relation between $T_{\text {dust }}$ and emissivity has values of $-0.5 \pm 0.2 \times 10^{-4}$, $-1.1 \pm 0.3 \times 10^{-4}$, and $-0.4 \pm 0.3 \times 10^{-4}$ in regions $\mathrm{A}, \mathrm{B}$, and $\mathrm{C}$, respectively. Thus, the value of the slope increases as the temperature decreases. For interclump dust, the predicted slope has a value of $-1.0 \pm 0.4 \times 10^{-4}$, close to the observed value of $-1.2 \times 10^{-4}$.

The vectors in Figs. 4 and 5 show the direction and magnitude (1- $\sigma$ standard deviation) of the movement of datapoints caused by the inverse relation between $T_{\text {dust }}$ and emissivity, when taking all the error sources of Table 2 into accout.

\section{Appendix D: Existence of emission from very small grains in non equilibrium}

Because the abundance of very small grains (VSGs) is enhanced in regions $\mathrm{A}$ and $\mathrm{C}$, we have to investigate whether there is any significant emission from these transiently heated grains at $100 \mu \mathrm{m}$. We assume that $100 \mu \mathrm{m}$ is the longest wavelength that may be affected by non-equilibrium grains. This is supported by the observations of L1780 (Ridderstad et al. 2006), which is a high-latitude cloud similar to L1642, and the translucent/cirrus cloud as studied by Laureijs et al. (2000). In both these clouds, the intensities at $100 \mu \mathrm{m}, 120 \mu \mathrm{m}, 150 \mu \mathrm{m}$, and $200 \mu \mathrm{m}$ are all well-fitted with a single black-body function, even in the positions where $12 \mu \mathrm{m}$ and $60 \mu \mathrm{m}$ emission have their maximum

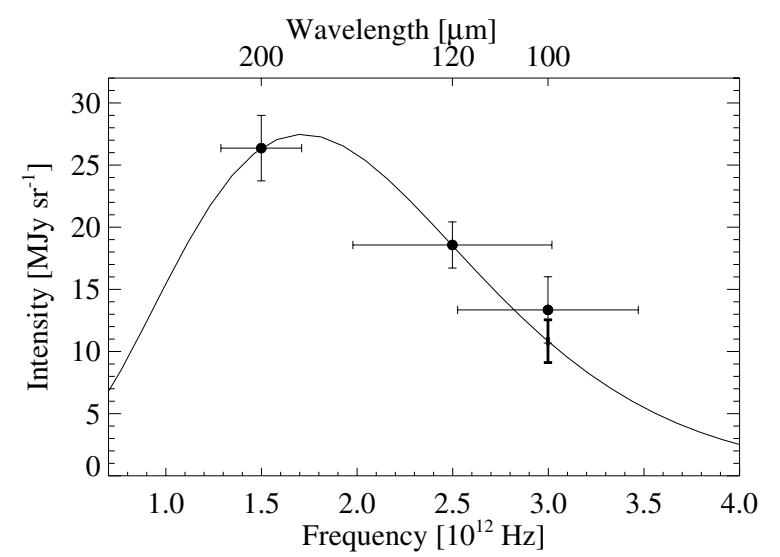

Fig. D.1. The intensities measured at a position inside region $\mathrm{C}$, at $100 \mu \mathrm{m}, 120 \mu \mathrm{m}$, and $200 \mu \mathrm{m}$. The solid curve is a modified black-body with a $v^{2}$ emissivity, fitted to the $120 \mu \mathrm{m}$ and $200 \mu \mathrm{m}$ data points. The thick vertical errors at $100 \mu \mathrm{m}$ show the $1-\sigma$ uncertainty of the fitted black-body, assuming $10 \%$ error for the $120 \mu \mathrm{m}$ and $200 \mu \mathrm{m}$ intensities. The thin vertical bars show the estimated errors of the observed intensities, $10 \%$ for the ISO data at $120 \mu \mathrm{m}$ and $200 \mu \mathrm{m}$, and $20 \%$ for the IRAS data at $100 \mu \mathrm{m}$. The horizontal bars show the bandwidth of each filter

values. On the other hand, at $80 \mu \mathrm{m}$ there is a significant excess over the black-body emission from thermal equilibrium grains.

In Fig. D. 1 we plot $120 \mu \mathrm{m}$ and $200 \mu \mathrm{m}$ intensities at the position in region $\mathrm{C}$, together with a modified black-body function fitted to them and an estimated error of the black-body function at $100 \mu \mathrm{m}$. When taking the errors into account, there is no evidence of excess emission at $100 \mu \mathrm{m}$. We believe that any excess emission in region $\mathrm{A}$ is even smaller, because region $\mathrm{A}$ is colder than region $\mathrm{C}$.

The $120 \mu \mathrm{m}$ and $200 \mu \mathrm{m}$ points in Fig. D. 1 are from ISO data, the $100 \mu \mathrm{m}$ point is from IRAS data (scaled to DIRBE calibration as in Lehtinen et al. 2004). The ON-source position in region $\mathrm{C}$ is at coordinates $\mathrm{RA}(\mathrm{J} 2000)=4^{\mathrm{h}} 36^{\mathrm{m}} 7.2^{\mathrm{s}}$, $\operatorname{Dec}(\mathrm{J} 2000)=-14^{\circ} 36^{\prime} 3^{\prime \prime}$. From the ON-source (ISO TDT numbers 83901895, 83901887) intensities, we have subtracted the mean values of intensities at two reference positions, located at coordinates $\mathrm{RA}(\mathrm{J} 2000)=4^{\mathrm{h}} 33^{\mathrm{m}} 17.3^{\mathrm{s}}$, $\operatorname{Dec}(\mathrm{J} 2000)=-15^{\circ} 58^{\prime} 12^{\prime \prime}$ (ISO TDT numbers 83901881, $83901882,83901890,83901889,83901885,83901893)$, and $\operatorname{RA}(\mathrm{J} 2000)=4^{\mathrm{h}} 28^{\mathrm{m}} 59.4^{\mathrm{s}}, \operatorname{Dec}(\mathrm{J} 2000)=-15^{\circ} 00^{\prime} 11^{\prime \prime}$ (ISO TDT numbers 83901896,83901888$)$. 